\title{
CT-Based Skeletal Muscle Loss for Predicting Poor Survival in Patients With Hepatocellular Carcinoma Experiencing Curative Hepatectomy Plus Adjuvant Transarterial Chemoembolization:a Preliminary Retrospective Study
}

\section{Siwei Yang}

Beijing Friendship Hospital

Zhiyuan Zhang

Beijing Friendship Hospital

Tianhao Su

Beijing Friendship Hospital

Jianan Yu

Beijing Friendship Hospital

Shasha Cao

Beijing Friendship Hospital

\section{Haochen Wang}

Beijing Friendship Hospital

Long Jin ( $\square$ longerg@hotmail.com)

Beijing Friendship Hospital

\section{Research Article}

Keywords: Skeletal muscle index, Hepatocellular carcinoma, Curative hepatectomy, Adjuvant transarterial chemoembolization, Prognosis

Posted Date: January 19th, 2022

DOI: https://doi.org/10.21203/rs.3.rs-1252322/v1

License: (c) (i) This work is licensed under a Creative Commons Attribution 4.0 International License. Read Full License 


\section{Abstract}

Background To evaluate the prognostic value of skeletal muscle index (SMI) and its change in patients with hepatocellular carcinoma (HCC) experiencing curative hepatectomy plus adjuvant transarterial chemoembolization (TACE).

Methods and Methods A total of 62 patients with HCC who underwent adjuvant TACE after curative hepatectomy were analysed retrospectively. Skeletal muscle area at the third lumbar level was quantitated using computed tomography images and was normalized for height squared to obtain skeletal muscle index (SMI). Skeletal muscle loss (SML) over six months was computed with two SMIs before and after hepatectomy plus adjuvant TACE. Correlation analyses were preformed to investigate factors associated with SML. The curves of cause-specific survival (CSS) were analysed using the Kaplan-Meier method. A Cox proportional hazards model was used to assess prognostic factors.

Results Low SMI was diagnosed in 23(37.1\%) patients preoperatively. The median SML standardized by 6 months was $-1.6 \%$ in the entire cohort. Liver cirrhosis and microvascular invasion correlated negatively with SML respectively $(\mathrm{r}=-0.320, P=0.002 ; \mathrm{r}=-0.243, P=0.021)$. Higher SML $(<-2.42 \%)$ predicted a significant reduction in CSS $(P=0.001)$, whereas low SMI did not $(P=0.687)$. Following the multivariate analysis for CSS, AFP> $400 \mathrm{ng} / \mathrm{ml}(\mathrm{HR}, 5.643 ; 95 \% \mathrm{Cl}$, 3.608-17.833; $P<0.001)$ and $S M L<-2.42 \%(H R, 6.586 ; 95 \% \mathrm{Cl}, 3.610-22.210 ; P<0.001)$ were independent predictors for poor CSS.

Conclusions Skeletal muscle loss during hepatectomy plus adjuvant TACE was remarkable. Higher SML was an independent risk factor for CSS in patients with HCC, especially those with liver cirrhosis.

\section{Introduction}

Malnutrition is usually observed in patients with chronic liver disease (CLD) and is caused by an imbalance of protein synthesis and breakdown resulting from abnormal liver synthesis, metabolism, detoxification and immune function[1]. However, due to fluid overload or obesity, malnutrition risk evaluation is often overlooked. In fact, malnutrition has recently been highlighted in clinical practice for CLD patients. As a new entity in the International Classification of Diseases, sarcopenia evaluated by low skeletal muscle index (SMI) at radiologic images and grip strength loss has become of increasing importance in clinical management and survival assessment for patients with hepatocellular carcinoma (HCC) $[2,3]$.

Currently, only performance status and serum albumin contribute to Child-Pugh and MELD scoring as indicators of nutritional condition for HCC patients with CLD, especially in the context of cirrhosis. Since patients with resectable $\mathrm{HCC}$ in the real world prone to be classified as having no or low risk owing to well liver function reserve, the differentiation value of some malnutrition screening tools, such as the NRS-2002 or royal-free hospital nutritional prioritization tool, is limited.

SMI measured by radiologic images reflecting skeletal muscle mass has superior accessibility and repeatability in evaluating the quantity of skeletal muscle for HCC patients[4]. Computed tomography (CT) and magnetic resonance imaging were routinely used in the diagnosis of HCC and tumor surveillance in follow-up. The latest review summarized that low SMI or sarcopenia was associated with a poor prognosis for HCC patients in different tumor stages who received liver resection or systemic therapy[5]. As noted, the HCC patients at early stage tend to have better liver function reserve, lower tumor burden and nonsignificant weight loss during treatment, and they recovered 
their weight quickly after diet adjustment. Therefore, the occurrence of sarcopenia and the loss of skeletal muscle mass during treatment in such patients may be overlooked extensively.

In the light of positive impact of postoperative adjuvant transarterial chemoembolization (TACE) on prognosis of HCC patients received curative hepatectomy, which was validated in two randomized controlled trials[6, 7], this combined therapy was recommended for the HCC patients at high risk of recurrence in the latest guidelines of primary liver cancer in China[8]. However, Kobayashi, A.et al.[9] showed the skeletal muscle mass is depleted significantly after hepatectomy, and repetitive platinum-based chemotherapy may also yield a negative impact[10-12]. To date, little is known about the prognostic value of SMI or related measurements for patients with HCC experiencing curative hepatectomy plus adjuvant TACE. Herein, this study retrospectively investigated whether the stratification of these HCC patents using skeletal muscle index or related parameters could identify subgroups with different survival risks.

\section{Materials And Methods}

\section{Patients Selection}

Patients with HCC who underwent adjuvant TACE after curative hepatectomy from January 2015 to December 2020 were consecutively and retrospectively analysed. The presence of at least one high risk characteristic including maximum tumor diameter $>5 \mathrm{~cm}$, tumor microvascular invasion (MVI), multiply tumors and liver cirrhosis was indication to preform adjuvant TACE since 2014 in our institution. Inclusion criteria were as follows $\mathbb{\|}$ curative hepatectomy preformed as initial treatment and HCC diagnosed pathologically; 邓the presence of at least one high risk characteristic of poor prognosis; 邓postoperative adjuvant TACE conducted at least once. Exclusion criteria were as

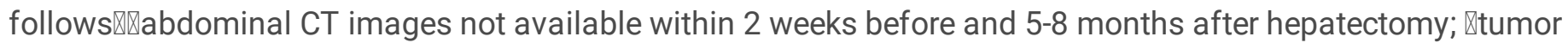
recurrence found between two CT examinations; 『a history of other malignancies; 邓patients with missing data or loss of follow-up. The flowchart of this study is shown in Figure 1. The study procedures conformed to the ethical guidelines of the Declaration of Helsinki, and the institutional review board approved this retrospective study. The requirement for informed consent of recruitment was waived.

\section{Hepatectomy and Postoperative Adjuvant TACE}

Patients with Barcelona Clinic Liver Cancer Group (BCLC) 0 or A and selective BCLC B stage HCC were candidates for curative hepatectomy referring to all tumor lesions found by preoperative imaging and intraoperative exploration. R0 resection (negative tumor involvement of margin in specimen) was confirmed histologically. Patients with BCLC B stage HCC, whose lesions were located at the same segment or lobe of liver and residual liver volume was sufficient, were considered to be eligible for surgery.

The first TACE was performed 3-4 weeks after hepatectomy. According to the laboratory results and performance status, TACE was conducted again after 4 weeks. Considering that Chinese guideline recommendation and protection of liver function reserve, no more than 3 times TACE sessions were conducted. Hepatic angiography was performed to clarify the liver feeding artery. Chemoemboliztion, including infusion of $50 \mathrm{mg} / \mathrm{m}^{2}$ oxaliplatin, $250 \mathrm{mg} / \mathrm{m}^{2}$ fluorouracil, $100 \mathrm{mg} / \mathrm{m}^{2}$ calcium leucovorin and embolization of $2-5 \mathrm{ml}$ iodized oil, was performed through the left and right hepatic arteries unselectively.

\section{SMI Quantitation}

Mimics Research software (Version 19.0, Materialise NV, Leuven, Belgium) was applied to calculate the targeted skeletal muscle area on preoperative and postoperative CT cross-sectional images at the level of the third lumbar 
vertebra level (L3), including psoas major, erector spinae, quadratus lumborum, transverse abdominis, internal and external oblique and rectus abdominis. The standard Hounsfield units-derived measurement on CT image as reference methods for quantifying skeletal muscle was proposed by Mitsiopoulos, N. et al[13]. Accordingly, the CT images were segmented by density thresholds ranging from -29 to 150 Hounsfield units, and boundaries of the targeted area were manually adjusted as needed (Figure 2). SMI was derived from the formula: skeletal muscle area $\left(\mathrm{cm}^{2}\right) /$ height $^{2}\left(\mathrm{~m}^{2}\right)$.

\section{Definitions and Follow-ups}

Liver cirrhosis was confirmed by histopathology of the liver parenchyma adjacent to the tumor. Major and minor hepatectomy were defined as the resection of $\geq 1$ or $<1$ hepatic segments, respectively. The adverse events were categorized as minor and major classifications according to whether the presence of additional therapy, prolonged hospitalization and severe consequences or not[14]. The criteria of low SMI were $42 \mathrm{~cm}^{2} / \mathrm{m}^{2}$ for men and $38 \mathrm{~cm}^{2} / \mathrm{m}^{2}$ for women recommended by the working group for creation of sarcopenia assessment criteria of Japan Society of Hepatology[15]. Multiphase CT and laboratory tests were performed 1 month after resection, every 3 months thereafter. The quantitative assessment of SMI change was obtained using the following formula: $\mathrm{SML}=$ [(postoperative SMI-preoperative SMI)×180/interval days between CT examinations] /pre- operative SMI $\times 100 \%$. The endpoint was cause-specific survival (CSS), defined as the interval between hepatectomy and the date of the last follow-up (30th April, 2021) or death. The cause of death was HCC or liver disease-related events (i.e., liver failure, variceal bleeding or hepatic encephalopathy).

\section{Statistical Analysis}

Continuous variables expressed as the mean \pm standard deviation or median (interquartile range) were compared by two-sample $t$ tests or Mann-Whitney $U$ tests, and categorical variables expressed as number (percentage) were compared by $\chi 2$ test or Fisher's exact tests. Correlation analyses were performed using the Spearman or Kendall's tau$\mathrm{b}$ analysis. The optimal cut-off value of SML was obtained by the receiver operating characteristic (ROC) curve according to the Youden index related to the endpoints. Survival curves were generated by using the Kaplan-Meier method and analysed with the log-rank test. A Cox proportional hazard model was used to analyse prognostic factors of CSS. Univariable analyses were performed after proportional hazard verification, and variables with $P<0.1$ were included in multivariate analysis. $P<0.05$ was considered statistically significant. All statistical analyses were performed using SPSS statistics software (Version 26.0; IBM Corp., Armonk, NY) and R software (version 4.0.3.; http:// www.r-project.org/)

\section{Results}

\section{Patient Demographics}

A total of $62 \mathrm{HCC}$ patients were eligible to be reviewed in this study. The baseline demographic and clinical characteristics of all patients are listed in Table 1 and Appendix Table 1 according to different SML groups and SMI groups respectively. The optimal cut-off value of SML was $-2.42 \%$, as determined by ROC curve analysis. Compared with the lower $S M L$ group, patients in the higher $S M L$ group ( $S M L<-2.42 \%)$ had a higher frequency of liver cirrhosis $(P=0.005)$, tumor $\mathrm{MVI}(P=0.048)$ and neutrophil to lymphocyte ratio (NLR) level $(P=0.026)$, but fewer TACE $\operatorname{sessions}(P=0.031)$. Additionally, male patients with a higher baseline $S M I$ had more skeletal muscle loss $(P=0.021)$. In the entire cohort, liver cirrhosis was identified in 36(58.1\%) patients. Low SMI was diagnosed in 23(37.1\%) patients preoperatively. The median time between two CT examinations was 188(164-218) days. A total of 164 adjuvant TACE 
sessions were administrated and all adverse events related to interventional procedures were nonsignificant, mainly including embolism syndrome and mild decreasing appetite. Baseline Child-Pugh A class was observed in all participants, and there was no deterioration of Child-Pugh class after combined treatment.

\section{The impacts of SMI and SML on CSS}

The cumulative CSS at 1, 3 and 5 years was $100.0 \%, 85.8 \%$ and $67.0 \%$, respectively. Baseline SMI based on Japanese criteria was unable to stratify the liver-related mortality risk of patients ( $P=0.687$, Figure $3 a)$.

The follow-up times in lower and higher SML groups were 36.1(19.2-45.1) months and 21.6(17.4-32.8) months, respectively. During the follow-up, Four (10.0\%) out of the 40 patients in the lower SML group died, and the cumulative CSS at 1, 3 and 5 years was 100\%, 93.6\% and $82.6 \%$, respectively. Nine (40.9\%) out of the 22 patients in the higher SML group died, and the cumulative CSS at 1, 3 and 5 years was $100 \%, 71.4 \%$ and $38.1 \%$, respectively. The difference in CSSs between the two groups was statistically significant (median CSS: NA vs. 911.0 days, $P=0.001$, Figure 3b).

\section{Features of SML between patient subgroups}

The median SML standardized by 6 months was $-1.6 \%$ in the entire cohort. Correlation analyses were used to further clarify the potential relationship between SML and other characteristics, including clinical variables (sex, age, BMI, BCLC class, tumor number, maximum tumor diameter, tumor differentiation, MVI, cirrhosis and TACE sessions) as well as routine laboratory values (TBIL, ALT, AST, PLT, AFP and NLR). Only two factors, the presence of cirrhosis $(r=-0.320, P=0.002)$ and $\mathrm{MVI}(r=-0.243, P=0.021)$, were negatively correlated with SML. Besides, patients with cirrhosis had a higher SML than those without cirrhosis (median SML: $-2.52 \%$ vs $0.69 \%, P=0.003$, Figure $4 a$ ). Likewise, a higher SML was seen in the patients with MVI than those without MVI (median SML: $-3.32 \%$ vs $-0.90 \%, P=0.016$, Figure $4 \mathrm{~b}$ ). When the cohort was divided into different subgroups according to NLR level categorized by median value or TACE sessions (>2), there was no significant differences in SML between different subgroups, respectively $(P=0.746 ; P=0.081)$.

\section{Association between lower SML and poor CSS}

Subgroups analyses were performed as per imbalanced variables between lower and higher SML groups, thereby minimizing the effects of confounding factors. As exploratory analyses showed, the higher SML was associated with reduced CSS in all subgroups stratified by the presence of cirrhosis or MVI (cirrhosis subgroup: $P=0.016$, Figure 5a; non-MVI subgroup: $P=0.005$, Figure $5 \mathrm{~d}$ ), despite with an equivocal difference in the non-cirrhosis subgroup and $\mathrm{MVI}$ subgroup ( $P=0.319$, Figure $5 b ; P=0.110$, Figure $5 c)$.

Multivariate analysis for CSS identified AFP $>400 \mathrm{ng} / \mathrm{mL}$ (HR:5.643, 95\%Cl:3.608-17.833, $P<0.001)$ and SML $<-2.42 \%$ (HR:6.586,95\%Cl:3.610-22.210, $P<0.001$ ) were independently predictive of poor CSS (Table 2).

\section{Discussion}

In this retrospective study, the skeletal muscle index of HCC patients experiencing curative hepatectomy plus adjuvant TACE significantly decreased over 6 months. More skeletal muscle mass loss served as an independent predictor of poor survival for HCC patients.

In advanced HCC patients, low SMI or sarcopenia was more common and proved to have a negative impact on these patients undergoing systemic therapy or intra-arterial therapy[16-18]. Indeed, the incidence of low SMI or sarcopenia 
in HCC patients who received curative treatments was also high and worthy of attention. In this study, low SMI patients accounted for $37.1 \%$ of study subjects, which was similar to $14.0-40.3 \%$ reported in previous studies[19-22]. Comparing to distribution of age or sex and the frequency of liver cirrhosis or chronic liver disease among these studies, it seems that the prevalence of low SMI was in accordance with liver disease progression. Besides, the effect of tumor burden led to reduced SMI due to decreasing appetite, increasing protein decomposition and aggravation of systemic inflammatory response[18, 23].

In addition, some prior studies also identified either low SMI or sarcopenia as indicative of recurrence and reduced survival for HCC patients undergoing hepatectomy[19, 24-26]. However, this study indicated that preoperative SMI did not stratify liver-related mortality risk, which could be a result of different endpoint and additional adjuvant TACE treatment.

When the presence of risk factors determined after curative hepatectomy for HCC patients, the combined therapy, namely curative hepatectomy plus adjuvant TACE, is an appropriate therapy. However, there were no studies elucidating the impact of skeletal muscle loss on such patients previously. As an integrated course of treatment aiming to achieve curative effect, skeletal muscle mass depletion should be quantified during a period of combined treatment rather than hepatectomy alone. Hence, the potential impact of TACE on skeletal muscle mass was considered in our study.

Our data showed that the median SML over 6 months was $-1.6 \%$ in the cohort of patients with Child-Pugh A class. A study found that the annual change rate in skeletal muscle mass at the level of L3 in counterparts was -1.3\%[27]. This demonstrated that SML during hepatectomy plus adjuvant TACE was also significant. In a study concerning changes in body composition for HCC patients underwent hepatectomy, the psoas muscle index was measured at different time points after surgery and measurements failed to exceed preoperative level in the following two years[9]. It is surmised that the loss of skeletal muscle mass caused by hepatectomy could last for a quite long time. Notably, skeletal muscle loss varied individually in this study. This could be attributed to the heterogeneity of individual liver function reserve and adjuvant TACE treatment.

Increasing evidence has demonstrated that more skeletal muscle loss may be related to poor tumor characteristics, such as MVI, larger tumor size and worse liver function, but not the treatment itself. Our results showed more skeletal muscle mass loss during treatment was associated with cirrhosis, MVI and inflammatory status, which is in line with the findings of other studies, and a negative correlation was observed between SML and liver cirrhosis as well as MVI. Recently, researchers have found that there was a distinct decrease in skeletal muscle mass per TACE cycle, and the degree of skeletal muscle mass depletion was a risk factor for overall survival[10,11]. They further proposed that the SML after the first TACE largely reflected worse tumor characteristics, and the liver function reserve was the main factor affecting SML in subsequent TACE cycles. Similarly, a study enrolling HCC patients who underwent radiotherapy to the liver demonstrated that HCC patients newly diagnosed with sarcopenia after radiotherapy had more Child-Pugh B or C as well as a larger tumor burden[28]. Another report including 603 patients with cirrhosis identified a higher NLR level in patients with severe sarcopenia than those in non- and sarcopenic groups[29]. In addition, Voron, T. et al.[19] thought sarcopenia was associated with HCC satellite lesions and MVI.

This study revealed that SML could evidently differentiate survival stratification. In spite of no statistical significance in some subgroups, it is probably related to the limited sample size. In the comparison of SMI, skeletal muscle mass loss and its rate may be more likely to reflect the response of patient's condition to therapeutic intervention. A cohort study also showed that the rate of muscle loss was a robust predictor for mortality in patients with cirrhosis instead of other single muscle mass-related measurements[30]. 
Our results showed that $S M L<-2.42 \%$ remained a significant independent risk factor for reduced CSS, indicating that the effect of skeletal muscle loss on prognosis was not the result of concomitant presence of adverse factors but rather the progression of liver dysfunction and worse general clinical condition. A mounting number of studies have unveiled that skeletal muscle mass loss was associated with pathophysiological alternations in the body, including decreased hepatic glycogen synthesis, hyperammonemia, glycogenolysis, myostatin, autophagy, and proinflammatory cytokines as well as endocrine changes[1, 31, 32]. However, more explorations are required in the underlying mechanisms.

To date, there is only general guidance for the prevention and treatment of sarcopenia $\varangle$ without a distinct role on decision-making in clinical settings. Our study suggested individual skeletal muscle loss and its rate were quite remarkable, especially for HCC patients with cirrhosis. Accordingly, it is necessary to increase awareness of declining skeletal muscle index during radiologic follow-up. If obvious skeletal muscle loss is observed, nutrition- supporting interventions, such as late evening snack, branched-chain amino acid supplementation and in-hospital exercise, should be considered[33-35].

The limitation of this study should be recognized. First, the data were retrospectively collected and analysed from a single center. Second, due to a lack of skeletal muscle function evaluation, sarcopenia could not be defined strictly, but the loss of skeletal muscle mass could be precisely quantified[36]. Third, because of the limited sample size of certain subgroup, some conclusions need to be further validated.

\section{Conclusion}

In conclusion, skeletal muscle loss during curative hepatectomy and adjuvant therapy has a detrimental impact on liver-related survival for patients with HCC, and seems to indicate poor liver and tumor characteristics.

\section{Abbreviations}

skeletal muscle index (SMI); skeletal muscle loss (SML); hepatocellular carcinoma (HCC); chronic liver disease (CLD); transarterial chemoembolization (TACE); cause-specific survival (CSS); Computed tomography (CT); microvascular invasion (MVI); Barcelona Clinic Liver Cancer Group (BCLC); receiver operating characteristic (ROC); neutrophil to lymphocyte ratio (NLR); the third lumbar vertebra level (L3)

\section{Declarations}

Ethics approval and consent: this retrospective study gained the approval from Capital Medical University affiliated Beijing Friendship Hospital's institutional review board in China. The registration number is 2021-P2-272-01.

Consent for publication: not applicable.

Data availability statement: The datasets generated or analyzed during the study are not publicly available due to the data of DICOM including patients' intimate information but are available from the corresponding author on reasonable request.

\section{Competing interests: none.}

Funding: none.

Author contributions

Page $7 / 13$ 
Siwei Yang, Zhiyuan Zhang proposed the conception and design; Siwei Yang, Zhiyuan Zhang, Tianhao Su conduted the development of methodology; Siwei Yang, Jianan Yu, Shasha Cao, Haochen Wang preformed the analysis and interpretation of data; Siwei Yang, Zhiyuan Zhang, Long Jin preformed the writing, review, and revision of the manuscript; Long jin assisted in the administration, or material support.

Acknowledgments: Not applicable.

\section{References}

1. Ebadi M, Bhanji RA, Mazurak VC, Montano-Loza AJ. Sarcopenia in cirrhosis: From pathogenesis to interventions. Journal of gastroenterology 2019;54:845-859

2. Plauth M, Bernal W, Dasarathy S, Merli M, Plank LD, Schütz T et al. Espen guideline on clinical nutrition in liver disease. Clinical nutrition (Edinburgh, Scotland) 2019;38:485-521

3. Buchard B, Boirie Y, Cassagnes L, Lamblin G, Coilly A, Abergel A. Assessment of malnutrition, sarcopenia and frailty in patients with cirrhosis: Which tools should we use in clinical practice? Nutrients 2020;12

4. Hiraoka A, Aibiki T, Okudaira T, Toshimori A, Kawamura T, Nakahara H et al. Muscle atrophy as pre-sarcopenia in japanese patients with chronic liver disease: Computed tomography is useful for evaluation. Journal of gastroenterology 2015;50:1206-1213

5. Marasco G, Serenari M, Renzulli M, Alemanni LV, Rossini B, Pettinari I et al. Clinical impact of sarcopenia assessment in patients with hepatocellular carcinoma undergoing treatments. Journal of gastroenterology 2020;55:927-943

6. Wang Z, Ren Z, Chen Y, Hu J, Yang G, Yu L et al. Adjuvant transarterial chemoembolization for hbv-related hepatocellular carcinoma after resection: A randomized controlled study. Clinical cancer research : an official journal of the American Association for Cancer Research 2018;24:2074-2081

7. Wei W, Jian PE, Li SH, Guo ZX, Zhang YF, Ling YH et al. Adjuvant transcatheter arterial chemoembolization after curative resection for hepatocellular carcinoma patients with solitary tumor and microvascular invasion: A randomized clinical trial of efficacy and safety. Cancer communications (London, England) 2018;38:61

8. Zhou J, Sun H, Wang Z, Cong W, Wang J, Zeng M et al. Guidelines for the diagnosis and treatment of hepatocellular carcinoma (2019 edition). Liver cancer 2020;9:682-720

9. Kobayashi A, Kaido T, Hamaguchi Y, Okumura S, Taura K, Hatano E et al. Impact of postoperative changes in sarcopenic factors on outcomes after hepatectomy for hepatocellular carcinoma. Journal of hepato-biliarypancreatic sciences 2016;23:57-64

10. Kobayashi T, Kawai H, Nakano O, Abe S, Kamimura H, Sakamaki A et al. Rapidly declining skeletal muscle mass predicts poor prognosis of hepatocellular carcinoma treated with transcatheter intra-arterial therapies. BMC cancer 2018;18:756

11. Fujita M, Takahashi A, Hayashi M, Okai K, Abe K, Ohira H. Skeletal muscle volume loss during transarterial chemoembolization predicts poor prognosis in patients with hepatocellular carcinoma. Hepatology research : the official journal of the Japan Society of Hepatology 2019;49:778-786

12. Dhooge M, Coriat R, Mir O, Perkins G, Brezault C, Boudou-Rouquette P et al. Feasibility of gemcitabine plus oxaliplatin in advanced hepatocellular carcinoma patients with child-pugh b cirrhosis. Oncology 2013;84:32-38

13. Mitsiopoulos N, Baumgartner RN, Heymsfield SB, Lyons W, Gallagher D, Ross R. Cadaver validation of skeletal muscle measurement by magnetic resonance imaging and computerized tomography. Journal of applied physiology (Bethesda, Md. : 1985) 1998;85:115-122

Page 8/13 
14. Gaba RC, Lewandowski RJ, Hickey R, Baerlocher MO, Cohen El, Dariushnia SR et al. Transcatheter therapy for hepatic malignancy: Standardization of terminology and reporting criteria. Journal of vascular and interventional radiology : JVIR 2016;27:457-473

15. Nishikawa H, Shiraki M, Hiramatsu A, Moriya K, Hino K, Nishiguchi S. Japan society of hepatology guidelines for sarcopenia in liver disease (1st edition): Recommendation from the working group for creation of sarcopenia assessment criteria. Hepatology research : the official journal of the Japan Society of Hepatology 2016;46:951963

16. Hiraoka A, Hirooka M, Koizumi Y, Izumoto H, Ueki H, Kaneto $M$ et al. Muscle volume loss as a prognostic marker in hepatocellular carcinoma patients treated with sorafenib. Hepatology research : the official journal of the Japan Society of Hepatology 2017;47:558-565

17. Hiraoka A, Kumada T, Kariyama K, Tada T, Tani J, Fukunishi S et al. Clinical importance of muscle volume in lenvatinib treatment for hepatocellular carcinoma: Analysis adjusted with inverse probability weighting. Journal of gastroenterology and hepatology 2020

18. Loosen SH, Schulze-Hagen M, Bruners P, Tacke F, Trautwein C, Kuhl C et al. Sarcopenia is a negative prognostic factor in patients undergoing transarterial chemoembolization (tace) for hepatic malignancies. Cancers 2019;11

19. Voron T, Tselikas L, Pietrasz D, Pigneur F, Laurent A, Compagnon P et al. Sarcopenia impacts on short- and longterm results of hepatectomy for hepatocellular carcinoma. Annals of surgery 2015;261:1173-1183

20. Harimoto N, Shirabe K, Yamashita YI, Ikegami T, Yoshizumi T, Soejima Y et al. Sarcopenia as a predictor of prognosis in patients following hepatectomy for hepatocellular carcinoma. The British journal of surgery 2013;100:1523-1530

21. Hamaguchi Y, Kaido T, Okumura S, Kobayashi A, Shirai H, Yao S et al. Preoperative visceral adiposity and muscularity predict poor outcomes after hepatectomy for hepatocellular carcinoma. Liver cancer 2019;8:92-109

22. Harimoto N, Yoshizumi T, Shimokawa M, Sakata K, Kimura K, Itoh S et al. Sarcopenia is a poor prognostic factor following hepatic resection in patients aged 70 years and older with hepatocellular carcinoma. Hepatology research : the official journal of the Japan Society of Hepatology 2016;46:1247-1255

23. Arends J, Baracos V, Bertz H, Bozzetti F, Calder PC, Deutz NEP et al. Espen expert group recommendations for action against cancer-related malnutrition. Clinical nutrition (Edinburgh, Scotland) 2017;36:1187-1196

24. Yabusaki N, Fujii T, Yamada S, Suzuki K, Sugimoto H, Kanda M et al. Adverse impact of low skeletal muscle index on the prognosis of hepatocellular carcinoma after hepatic resection. International journal of surgery (London, England) 2016;30:136-142

25. Kamachi S, Mizuta T, Otsuka T, Nakashita S, Ide Y, Miyoshi A et al. Sarcopenia is a risk factor for the recurrence of hepatocellular carcinoma after curative treatment. Hepatology research : the official journal of the Japan Society of Hepatology 2016;46:201-208

26. Xu L, Jing Y, Zhao C, Zhang Q, Zhao X, Yang J et al. Preoperative computed tomography-assessed skeletal muscle index is a novel prognostic factor in patients with hepatocellular carcinoma following hepatectomy: A meta-analysis. Journal of gastrointestinal oncology 2020;11:1040-1053

27. Hanai T, Shiraki M, Ohnishi S, Miyazaki T, Ideta T, Kochi T et al. Rapid skeletal muscle wasting predicts worse survival in patients with liver cirrhosis. Hepatology research : the official journal of the Japan Society of Hepatology 2016;46:743-751

28. Lee J, Cho Y, Park S, Kim JW, Lee IJ. Skeletal muscle depletion predicts the prognosis of patients with hepatocellular carcinoma treated with radiotherapy. Frontiers in oncology 2019;9:1075 
29. Ebadi M, Bhanji RA, Dunichand-Hoedl AR, Mazurak VC, Baracos VE, Montano-Loza AJ. Sarcopenia severity based on computed tomography image analysis in patients with cirrhosis. Nutrients 2020;12

30. Welch N, Dasarathy J, Runkana A, Penumatsa R, Bellar A, Reen J et al. Continued muscle loss increases mortality in cirrhosis: Impact of aetiology of liver disease. Liver international : official journal of the International Association for the Study of the Liver 2020;40:1178-1188

31. Choi K, Jang HY, Ahn JM, Hwang SH, Chung JW, Choi YS et al. The association of the serum levels of myostatin, follistatin, and interleukin-6 with sarcopenia, and their impacts on survival in patients with hepatocellular carcinoma. Clinical and molecular hepatology 2020;26:492-505

32. Dasarathy S, Merli M. Sarcopenia from mechanism to diagnosis and treatment in liver disease. Journal of hepatology 2016;65:1232-1244

33. Chen CJ, Wang LC, Kuo HT, Fang YC, Lee HF. Significant effects of late evening snack on liver functions in patients with liver cirrhosis: A meta-analysis of randomized controlled trials. Journal of gastroenterology and hepatology 2019;34:1143-1152

34. Hiraoka A, Michitaka K, Kiguchi D, Izumoto H, Ueki H, Kaneto $M$ et al. Efficacy of branched-chain amino acid supplementation and walking exercise for preventing sarcopenia in patients with liver cirrhosis. European journal of gastroenterology \& hepatology 2017;29:1416-1423

35. Koya S, Kawaguchi T, Hashida R, Hirota K, Bekki M, Goto E et al. Effects of in-hospital exercise on sarcopenia in hepatoma patients who underwent transcatheter arterial chemoembolization. Journal of gastroenterology and hepatology 2019;34:580-588

36. Hiraoka A, Michitaka K, Ueki H, Kaneto M, Aibiki T, Okudaira T et al. Sarcopenia and two types of presarcopenia in japanese patients with chronic liver disease. European journal of gastroenterology \& hepatology 2016;28:940947

\section{Tables}

\section{Figures}

\section{Figure 1}

Flowchat shows patient selection.

\section{Figure 2}

Skeletal muscle loss marked by green mask over 171 days in a 52-year-old man with a single moderate differentiated HCC lesion. (a) Preoperative skeletal muscle index (SMI) on axial CT image at the L3 level was $46.97 \mathrm{~cm} 2 / \mathrm{m} 2$. (b) postoperative SMI at the same plane on CT image was $40.82 \mathrm{~cm} 2 / \mathrm{m} 2$.

\section{Figure 3}


Table 1 Baseline demographic and clinical characteristics in higher and lower SML groups variable $\quad$ Total $(\mathrm{n}=62) \quad$ higher SML $\quad(\mathrm{n}=22) \quad$ lower $\mathrm{SML}(\mathrm{n}=40) \quad P$

\begin{tabular}{|c|c|c|c|c|c|}
\hline \multicolumn{2}{|l|}{ Sex (male) } & $49(79.0)$ & 17(77.3) & $32(80.0)$ & 0.801 \\
\hline \multicolumn{2}{|l|}{ Age (year) } & $59.39 \pm 10.6$ & $57.3 \pm 11.2$ & $60.5 \pm 10.2$ & 0.258 \\
\hline \multirow{2}{*}{$\begin{array}{l}\text { BMI } \\
\left(\mathrm{kg} / \mathrm{m}^{2}\right)\end{array}$} & male & $24.1 \pm 3.4$ & $24.8 \pm 3.2$ & $23.7 \pm 3.5$ & 0.291 \\
\hline & female & $22.5 \pm 3.2$ & $21.8 \pm 1.7$ & $23.0 \pm 3.9$ & 0.557 \\
\hline \multirow{2}{*}{$\begin{array}{l}\text { SMl } \\
\left(\mathrm{kg}^{2} / \mathrm{m}^{2}\right)\end{array}$} & male & $45.0 \pm 6.9$ & $48.0 \pm 8.1$ & $43.3 \pm 5.6$ & 0.021 \\
\hline & female & $39.0 \pm 5.5$ & $39.5 \pm 8.4$ & $38.6 \pm 3.2$ & 0.820 \\
\hline \multirow[t]{2}{*}{ SML (\%) } & male & $-1.5 \pm 3.2$ & $-4.9 \pm 1.5$ & $0.38 \pm 2.1$ & $<0.001$ \\
\hline & female & $-1.4 \pm 3.2$ & $-4.9 \pm 1.3$ & $0.60 \pm 1.8$ & 0.002 \\
\hline \multicolumn{2}{|c|}{$\begin{array}{l}\text { Aetiology } \\
\text { (HBV/HCV/others) }\end{array}$} & $49(79.0) / 3(4.8) / 10(16.1)$ & 19(86.4)/0/3(13.6) & $30(75.0) / 3(7.5) / 7(17.5)$ & 0.473 \\
\hline \multicolumn{2}{|c|}{$\mathrm{BCLC}(0 / \mathrm{A} / \mathrm{B})$} & $6(9.7) / 53(85.5) / 3(4.8)$ & $4(18.2) / 16(72.7) / 2(9.1)$ & $2(5.0) / 37(92.5) / 1(2.5)$ & 0.109 \\
\hline \multicolumn{2}{|c|}{$\begin{array}{l}\text { Tumor number } \\
\text { (solitary/multiple) }\end{array}$} & $54(87.1) / 8(12.9)$ & 19(86.4)/3(13.6) & $35(87.5) / 5(12.5)$ & 0.898 \\
\hline \multicolumn{2}{|c|}{$\begin{array}{l}\text { Maximum tumor } \\
\text { diameter }(\mathrm{cm})\end{array}$} & $4.3 \pm 2.1$ & $3.9 \pm 2.3$ & $4.5 \pm 1.9$ & 0.278 \\
\hline \multicolumn{2}{|c|}{$\begin{array}{l}\text { Differentiation(well } \\
\text { or moderate/poor) }\end{array}$} & $54(87.1) / 8(12.9)$ & 18(81.8)/4(18.2) & $36(90.0) / 4(10.0)$ & 0.438 \\
\hline \multicolumn{2}{|c|}{$\begin{array}{l}\text { Hepatectomy } \\
\text { (major/minor) }\end{array}$} & $49(79.0) / 13(21.0)$ & 18(81.8)/4(18.2) & $31(77.5) / 9(22.5)$ & 0.756 \\
\hline \multicolumn{2}{|c|}{$\begin{array}{l}\text { MVI } \\
\text { (presence/absence) }\end{array}$} & $13(21.0) / 49(79.0)$ & $8(36.4) / 14(63.6)$ & $5(12.5) / 35(87.5)$ & 0.048 \\
\hline \multicolumn{2}{|c|}{$\begin{array}{l}\text { Cirrhosis } \\
\text { (presence/absence) }\end{array}$} & $36(58.1) / 26(41.9)$ & 18(81.8)/4(18.2) & $18(45.0) / 22(55.0)$ & 0.005 \\
\hline \multicolumn{2}{|c|}{$\begin{array}{l}\text { TACE sessions } \\
(1 / 2 / 3)\end{array}$} & $5(8.1) / 12(19.4) / 45(72.6)$ & $1(4.5) / 8(36.4) / 13(59.1)$ & $4(10.0) / 4(10.0) / 32(80.0)$ & 0.031 \\
\hline \multicolumn{2}{|c|}{$\begin{array}{l}\text { Child-Pugh class } \\
\text { (A/B) }\end{array}$} & $62(100.0)$ & $22(100.0) / 0$ & $40(100.0) / 0$ & - \\
\hline \multicolumn{2}{|c|}{ MELD-Na score } & 7.0(6.0 9.0) & $7.0(6.0 \sim 8.0)$ & 7.0(6.0 9.0) & 0.359 \\
\hline \multicolumn{2}{|l|}{$\operatorname{ALB}(g / L)$} & $40.0 \pm 3.8$ & $39.8 \pm 4.2$ & $39.7 \pm 3.6$ & 0.878 \\
\hline \multicolumn{2}{|c|}{ TBIL (umol/L) } & $15.5(11.9 \sim 22.6)$ & 14.3(11.6 17.0) & 16.8(13.1 24.7) & 0.064 \\
\hline \multicolumn{2}{|l|}{$\operatorname{ALT}(\mathrm{U} / \mathrm{L})$} & $23.0(17.0 \sim 31.3)$ & $24.5(15.0 \sim 30.3)$ & $22.0(18.0 \sim 39.3)$ & 0.466 \\
\hline \multicolumn{2}{|l|}{ AST (U/L) } & $29.2(22.6 \sim 36.6)$ & $26.5(21.0 \sim 32.5)$ & $30.7(23.1 \sim 36.9)$ & 0.406 \\
\hline \multicolumn{2}{|c|}{$\operatorname{PLT}\left(10^{9} / \mathrm{L}\right)$} & $159.4 \pm 59.4$ & $153.6 \pm 62.8$ & $162.3 \pm 58.0$ & 0.573 \\
\hline \multicolumn{2}{|c|}{ AFP (ng/ml) } & $20.7(2.7 \sim 305.9)$ & $53.6(2.6 \sim 325.4)$ & $15.0(2.8 \sim 297.9)$ & 0.953 \\
\hline \multicolumn{2}{|l|}{ NLR } & $1.9 \pm 1.0$ & $\begin{array}{l}2.3 \pm 1.1 \\
\text { Page } 11 / 13\end{array}$ & $1.7 \pm 0.9$ & 0.026 \\
\hline
\end{tabular}




\begin{tabular}{|c|c|c|c|c|}
\hline Variables & Univariate analysis & & Multivariate analysis & \\
\hline & $\mathrm{HR} \otimes 95 \% \mathrm{Cl} \rrbracket$ & $P$ value & $\mathrm{HR} \otimes 95 \% \mathrm{CI} \rrbracket$ & $P$ value \\
\hline Age (>60y) & $0.384(0.122 \sim 1.202)$ & 0.288 & & \\
\hline Sex (male) & $2.053(0.453 \sim 9.308)$ & 0.351 & & \\
\hline SML $(<-2.42 \%)$ & $5.761(1.761 \sim 18.848)$ & 0.004 & $6.586(3.610 \sim 22.210)$ & $<0.001$ \\
\hline $\mathrm{BMI}\left(>23 \mathrm{~kg} / \mathrm{m}^{2}\right)$ & $3.316(1.018 \sim 10.802)$ & 0.147 & & \\
\hline Maximum tumor diameter $(>5 \mathrm{~cm})$ & $1.429(0.466 \sim 4.384)$ & 0.532 & & \\
\hline tumor number (multiple) & $1.871(0.513 \sim 6.826)$ & 0.343 & & \\
\hline $\mathrm{MVI}$ & $1.163(0.319 \sim 4.238)$ & 0.819 & & \\
\hline Cirrhosis & $2.158(0.593 \sim 7.845)$ & 0.243 & & \\
\hline $\mathrm{BCLC}(\mathrm{B})$ & $2.582(0.571 \sim 11.688)$ & 0.218 & & \\
\hline TBIL (>17.1ummol/L) & $0.724(0.233 \sim 2.355)$ & 0.591 & & \\
\hline $\operatorname{ALB}(<35 \mathrm{~g} / \mathrm{L})$ & 3.333 (0.730 15.212) & 0.120 & & \\
\hline $\operatorname{ALT}(>40 \mathrm{U} / \mathrm{L})$ & $0.330(0.043 \sim 2.541)$ & 0.287 & & \\
\hline PLT $\left(<100 \times 10^{9} / \mathrm{L}\right)$ & $2.364(0.648 \sim 8.626)$ & 0.193 & & \\
\hline AFP (>400 ng/ml) & 4.478 (1.494 13.419) & 0.007 & 5.643 (3.608 17.833) & $<0.001$ \\
\hline TACE (>2 sessions) & $0.561(0.172 \sim 1.822)$ & 0.336 & & \\
\hline $\operatorname{NLR}\left(>1.83^{*}\right)$ & $1.127(0.378 \sim 3.364)$ & 0.830 & & \\
\hline
\end{tabular}

Comparisons of Survival curves in different SMI or SML subgroups. (a) Cumulative CSS curves between the baseline low and high SMI groups. (b) Cumulative CSS curves between the lower and higher SML groups.

\section{Figure 4}

(a) Boxplot diagram showed patients with cirrhosis had higher SML than those without cirrhosis (median SML: -2.52 $\%$ vs $0.69 \%, P=0.003$ ). (b) higher SML was observed in patients with MVI than those without MVI (median SML: -3.32 $\%$ vs $-0.90 \%, P=0.016)$.

\section{Figure 5}

Subgroup analyses for CSS between lower and higher SML groups. (a) Patients with higher SML had a reduced CSS in the patients with cirrhosis. (b) No significant difference was found in CSS between patients with lower and higher 
SML subgroups in non-cirrhosis patients. (c) A trend towards an impaired CSS was observed in patients with higher SML in patients with MVI.(d) Patients with higher SML had a reduced CSS in the patients with non-MVI.

\section{Supplementary Files}

This is a list of supplementary files associated with this preprint. Click to download.

- Appendixtable1.docx 